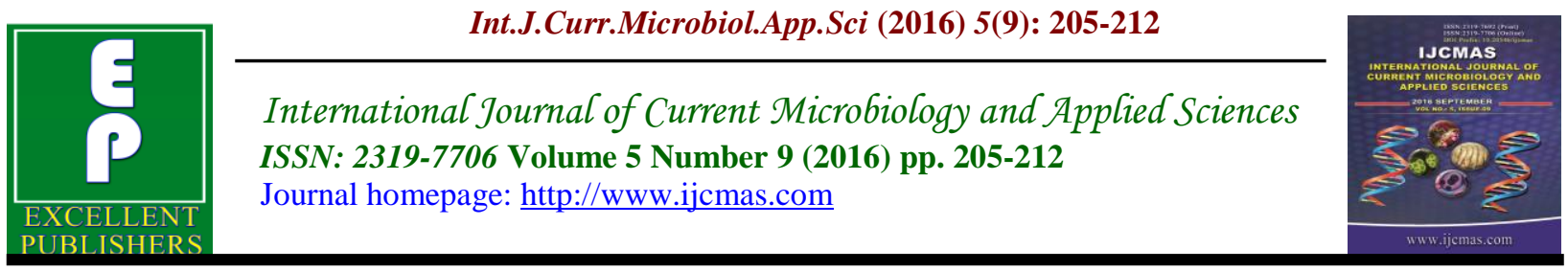

Original Research Article

http://dx.doi.org/10.20546/ijcmas.2016.509.023

\title{
Comparison of Susceptibility of Gram Negative Bacilli to Cephalosporins and Ciprofloxacin
}

\author{
Vishwajith*, K. Anuradha and D. Venkatesha \\ Department of Microbiology, Mysore Medical College and Research Institute, \\ Mysore - 570 001, India \\ *Corresponding author
}

\section{Keywords}

Cephalosporins, ciprofloxacin, gram negative bacilli, antibiotic susceptibility test.

\section{Article Info}

Accepted:

13 August 2016 Available Online: 10 September 2016

\section{A B S T R A C T}

Cephalosporins and ciprofloxacin are commonly used in the treatment of gram negative bacilli infections. Third generation cephalosporins are first choice but expensive than ciprofloxacin. So there is a need to compare their susceptibility. To compare the susceptibility of gram negative bacilli clinical isolates to cephalosporins and ciprofloxacin. Susceptibility of ESBL and AmpC producers to ciprofloxacin. Disk diffusion method performed on200 Gram negative bacilli isolates. Susceptibilityto cefuroxime, cefotaxime, ceftazidime, ceftazidime with clavulanic acid, cefoxitin, cefepime and ciprofloxacin were tested. ESBL detected by $>7 \mathrm{~mm}$ zone with ceftazidime clavulanic acid than ceftazidime alone. AmpC detected by cefoxitin resistant and cefepime sensitive. Pseudomonas isolates exudates 14 (29\%), burns $26(54 \%)$, bood $8(17 \%)$. Susceptibility to ciprofloxacin, cefepime in exudate $8(57 \%)$, $6(43 \%)$, burns $10(38 \%), 8(31 \%)$ respectively. Blood isolates susceptible to cefepime 6(75\%) other cephalosporins and ciprofloxacin4(50\%). Escherichia coli isolates 14 (28\%) exudates, 6(12\%) blood, $14(28 \%)$ sputum and 18(36\%)urine. Blood isolates $100 \%$ susceptibility to ciprofloxacin, cefepime Exudate 4(28\%),8(57\%). Equal susceptibility to both $8(57 \%)$ sputum. Urine $12(66 \%), 14(78 \%)$ respectively. Klebsiella $20(32 \%)$ exudates and burns, 20(32\%)sputum,4(6\%)blood. Their susceptibility to ciprofloxacin 4(22\%) 2(11\%),10(50\%) and cefepime 4(22\%), 4(22\%), 2(50\%), $10(50 \%)$ respectively. Enterobacter blood 12(50\%), exudate, burns sputum 4(17\%) each. Susceptibility to ciprofloxacin $4(100 \%)$ exudates and burns, $8(67 \%)$ blood. Resistance to cefepime seen in all except blood isolates 8(67\%). Citrobacter isolates from exudates $6(60 \%)$, from blood and urine2(20\%) each.Exudate2(33\%) sensitive to all cephalosporins and ciprofloxacin. Blood, urine1(100\%) sensitive to ciprofloxacin. Acinetobacter 6(100\%) exudates.2(33\%) sensitive only to cefepime Ciprofloxacin susceptibility seen in Pseudomonas 54\%, E.coli 72\%, Klebsiella 29\%, Enterobacter 66\%, Citrobacter $80 \%$ and Acinetobacter resistant and cefepime susceptibility 21\%, $60 \%, 25 \%, 41 \%, 40 \%$ and $33 \%$ respectively. Among 8 ESBL, 5 AmpC producers $4(50 \%)$ and 2(40\%) sensitive to ciprofloxacin respectively. Ciprofloxacin was found to be more active than the fourth generation cephalosporin cefepime against gram negative bacilli. Since ciprofloxacin are cheaper it reduces the treatment cost. 


\section{Introduction}

Gram-negative bacteria are common causes of intra-abdominal infections (IAIs), urinary tract infections (UTIs), nosocomial pneumonia, and bacteremia (Sievert et al., 2013). The most common organisms responsible for these infections are multidrug resistant gram negative bacilli particularly members of the family Enterobacteriaceae and non fermenting gram negative rods (Bhattacharjee et al., 2008).

Antimicrobial resistance among GNB is increasing worldwide. This is a major public health problem and a cause for both substantial morbidity and mortality among hospitalized patients. A direct correlation has been shown between resistance of GNB and patient mortality, cost of patient care and length of stay in the hospital (Shawn et al., 2007).

Cephalosporins and ciprofloxacin are commonly used in the treatment of these infections. Cephalosporins are similar to penicillins, but more stable to many bacterial $\beta$ lactamases and therefore have a broader spectrum of activity. However, strains of E coli and Klebsiella sp expressing extended-spectrum $\beta$ lactamases that can hydrolyze most cephalosporins are a growing clinical concern. Beside this Cephalosporins can cause hypersensitivity reactions that are identical to those of penicillins (Bertram).

Fluoroquinolones were originally developed because of their excellent activity against gram-negative aerobic bacteria they had limited activity against gram-positive organisms. Further ciprofloxacin are cheap, and its half-life is 3- 5 hours with a bioavailability of $70 \%$ it can be orally administered twice daily. It is widely distributed in body fluids and tissues and well tolerated compared to cephalosporins (Bertram).

In the Ambler structural classification of $\beta$ lactamases, AmpC enzymes belong to Class $\mathrm{C}$, while in the functional classification scheme of Bush these are assigned to group 3 (Ambler et al., 1980; Bush et al., 1995) . These enzymes can be chromosomal or plasmid encoded. AmpC $\beta$-lactamases are clinically significant, (Cordron et al., 2000) since these confer resistance to cephalosporins in the oxyimino group (cefotaxime, ceftazidime, ceftriaxone), 7- $\alpha$ methoxy cephalosporins (cefoxitin or cefotetan) and are not affected by available $\beta$-lactamase inhibitors (clavulanate, sulbactam, tazobactam) (Phillipon et al., 2002). Plasmid mediated AmpC $\beta$ lactamases differ from chromosomal AmpCs in being uninducible and are typically associated with broad multidrug resistance (Barnaud et al., 1998). Plasmid mediated AmpC $\beta$-lactamases are present in isolates of Klebsiella pneumoniae, $K$. oxytoca, Salmonella spp., Proteus mirabilis, Escherichia coli, Citrobacter freundii and Enterobacter aerogenes (Thomson et al., 2001). In E. coli, high level production of chromosomally mediated AmpC $\beta$-lactamases is also present (Black et al., 2005). The treatment options for infections caused by organisms expressing AmpC $\beta$-lactamases are limited.

Resistance to extended-spectrum cephalosporins in $E$. coli and, in particular, $K$. pneumoniae has become a worldwide problem (Paterson et al., 2005). Dissemination of ESBLproducing Enterobacteriaceae in the community poses a new threat, since this may become a powerful reservoir for the continued influx of resistant strains into hospitals (Ben-Ami et al., 2006; Pitout et al., 2005). 
Thus there is a need for detecting AmpC $\beta$ lactamases so as to avoid therapeutic failures. The reports of AmpC $\beta$-lactamases from India are still limited. Therefore, there is a need to study for both extended spectrum $\beta$-lactamase (ESBL) and AmpC $\beta$ lactamases.

Resistance to Fluoroquinolones and cephalosorins are increasing and there is a need to evaluate their resistance pattern. Keeping this in mind we decided to compare the susceptibility of gram negative bacilli clinical isolates to ciprofloxacin with that of second, third and fourth generation of cephalosorins.

The main objectives of this study to compare the susceptibility of gram negative bacilli clinical isolates to cephalosporins and ciprofloxacin.

Susceptibility of ESBL and AmpC producers to ciprofloxacin.

\section{Materials and Methods}

Gram negative bacterial isolates from samples of exudate, sputum, blood, urine and burn patients were identified using the conventional methods and tested for Antibiotic susceptibility testing by Kirby Bauer method using cefuroxime, cefotaxime, ceftazidime, ceftazidime with clavulanic acid, cefoxitin, cefepime and ciprofloxacin as per CLSI guidelines.

ESBL producers were detected by disc diffusion method using ceftazidime and ceftazidime/clavulanic acid disc as per CLSI guidelines. Ceftazidime-clavulanic acid disc was placed toward the center of the plate, a ceftazidime disc $(30 \mathrm{mg})$ was placed $15 \mathrm{~mm}$ out from the edge of ceftazidime-clavulanic acid disc at $90^{\circ}$ angles, so that it's inner edge was $15 \mathrm{~mm}$ from it. Plates were incubated at $35^{\circ} \mathrm{C}$, aerobically for $18-24 \mathrm{~h}$. Organism was detected as ESBL by $>7 \mathrm{~mm}$ zone with ceftazidime clavulanic acid than ceftazidime alone.

AmpC producers were detected by disc diffusion method using cefoxitin and cefepime discs. Cefoxitin zone of $<18 \mathrm{~mm}$ was taken as cefoxitin resistant. Isolates resistant to cefoxitin and sensitive to cefepime was taken as AmpC producers.

\section{Results and Discussion}

A total of 200 Gram negative bacilli isolated from various clinical specimens were included. Among them 62 isolates were from exudates samples, 48 from burns, 38 from sputum, 32 from blood and 20 from urine samples. (Table 1)

The organisms isolated are Klebsiella spp.62(31\%), Escherichia coli50 (25\%), Pseudomonas spp48 (24\%), Enterobacter spp24 (12\%), Citrobacter spp 10 (5\%), and Acinetobacter spp6(3\%).(Table 2)

Pseudomonas was isolated frequently from burn wounds, Enterobacter from blood while Klebsiella sppfrom exudate and respiratory tract, Escherichia coli from urine specimens.(Table3)

The susceptibility pattern of various isolates are shown in Table 4.All isolates showed good susceptibility to ciprofloxacin except Acinetobacter spp only one strain of it was sensitive to cefepime.

Among 100 isolates, 8 isolates were ESBL producers and 5 isolates were AmpC producers out of which $4(50 \%)$ isolates of ESBL and 2 isolates of AmpC producers were ciprofloxacin susceptible.

Klebsiella species isolates from exudates, burns and sputum showed susceptibility to 
ciprofloxacin $2(22 \%), 2(11 \%), 10(50 \%)$ and cefepime $4(22 \%), 2(50 \%), 10(50 \%)$ respectively.

Escherichia coli susceptibility to ciprofloxacin and cefepime, blood isolates showed $100 \%$ susceptibility whereas isolates from exudate showed $4(28 \%), 8(57 \%)$, from urine $12(66 \%), 14(78 \%)$ respectively. Equal susceptibility to both $8(57 \%)$ was observed in sputum isolates.

Pseudomonas species isolated from various specimens showed susceptibility to ciprofloxacin and cefepime from exudate 8 (57\%), 6(43\%), burns 10(38\%), 8(31\%) respectively. Blood isolates were susceptible to cefepime $6(75 \%)$ other cephalosporins and ciprofloxacin $4(50 \%)$.

Enterobacter species isolated from exudate and burns showed $100 \%$ susceptibility tociprofloxacin, $8(67 \%)$ of blood isolates were susceptible. Only 8(67\%) blood isolates were cefepime susceptible.

Citrobacter species isolated from blood, urine were $100 \%$ sensitive to ciprofloxacin. Exudate isolate2 (33\%) wassensitive to all cephalosporins and ciprofloxacin.

Acinetobacter species isolated from exudate alone. Out of which only 2(33\%) was sensitive only to cefepime. All other antibiotics were resistant.

Ciprofloxacin susceptibility seen in Pseudomonas 54\%, E.coli72\%, Klebsiella 29\%, Enterobacter 66\%, Citrobacter $80 \%$ and Acinetobacter resistant .

Bacterial resistance to ceftazidime is a serious problem. Approximately $40 \%$ ofEnterobacter cloacae were resistant to ceftazidime, according to studies of ICU isolates in the United States between 1987 and 1991 and between 1994 and 1995
(Archibald et al., 1997). In our study all Entrobacterspp were resistant to ceftazidime (Table 4). This is probably related to production of stably derepressed chromosomal class-1 $\beta$-lactamase, which hydrolyzes $\beta$-lactam antibiotics other than carbapenems. Previous use of thirdgeneration cephalosporins is more likely to cause the selection of resistance to $\beta$-lactams in blood isolates of Enterobacter species, which is associated with higher mortality.

An increased ciprofloxacin resistance among Enterobacter species was seen in Belgium and France compared with previous studies performed in 1990 (Verbist, 1991) and 1991 (Jarlier et al., 1996). In our study $66 \%$ of Enterobacter spp. was sensitive to ciprofloxacin.

In Portugal and France, 36\% and 26\%, respectively, of Klebsiella pneumoniae showed decreased susceptibility to ceftazidime. This was probably caused by the production of extended-spectrum $\beta$ lactamases (Livermore, 1995) and is concordant with a study by Livermore and Yuan. According to Jarlier et al., decreased susceptibility to ceftazidime among $K$ pneumoniae in French ICUs was 36\% in 1991. In the United States, resistance to ceftazidime among $K$ pneumoniae in ICU isolates increased from $3.6 \%$ in 1990 to $14.4 \%$ in 1993. In our study Klebsiella spp showed $6 \%$ sensitivity to ceftazidime.

The potency of cefepime, a parenteral aminothiazoly lmethoxyimino cephalosporin, was assessed against 256 ceftazidime-resistant Gram-negative bacilli from five medical centers in the United States. In addition, cefepime activity was compared with that of ciprofloxacin and imipenem against 506 ceftazidime-resistant Gram-negative bacilli collected during an 11-medical-center international study. All 
US clinical isolates were susceptible $(<$ or $=$ 8 micrograms $/ \mathrm{ml}$ ) to cefepime except Enterobacter cloacae (94\% susceptible) and Pseudomonas aeruginosa (19\% susceptible). Enterobacteriaceae isolates from the 11- nation sample were $>80 \%$ cefepime susceptible with the exception of those from Brazil (48\% susceptible) and Italy (55\% susceptible (Jones et al., 1994).

Table.1 Distribution of various clinical specimens

\begin{tabular}{|l|c|}
\hline Exudate & 62 \\
\hline Burns & 48 \\
\hline Sputum & 38 \\
\hline Blood & 32 \\
\hline Urine & 20 \\
\hline Total samples & 200 \\
\hline
\end{tabular}

Table.2 Isolates from various clinical specimens

\begin{tabular}{|l|c|}
\hline Klebsiella spp & 62 \\
\hline Escherichia coli & 50 \\
\hline Pseudomonas spp & 48 \\
\hline Enterobacter spp & 24 \\
\hline Citrobacter spp & 10 \\
\hline Acinetobacter spp & 6 \\
\hline Total samples & 200 \\
\hline
\end{tabular}

Table.3 Distribution of various isolates in different clinical specimens

\begin{tabular}{|l|c|c|c|c|c|}
\hline & Exudates & Burns & Sputum & Blood & Urine \\
\hline Pseudomonas & 14 & 26 & - & 8 & - \\
\hline E coli & 14 & - & 14 & 6 & 18 \\
\hline Klebsiella & 20 & 18 & 20 & 20 & - \\
\hline Enterobacter & 4 & 4 & 4 & 12 & - \\
\hline Citrobacter & 6 & - & - & 2 & 2 \\
\hline Acinetobacter & 6 & - & - & - & - \\
\hline
\end{tabular}

Table.4 Susceptibility pattern of Gram negative bacterial isolates

\begin{tabular}{|l|c|c|c|c|c|}
\hline Organism & Cefotaxime & Cefuroxime & Ceftazidime & Cefepime & Ciprofloxacin \\
\hline Pseudomonas & $4(8.3)$ & $4(8.3)$ & $10(21)$ & $26(54)$ & $30(62.5)$ \\
\hline E Coli & $6(12)$ & $6(12)$ & $6(12)$ & $30(60)$ & $36(72)$ \\
\hline Klebsiella & $4(6)$ & $4(6)$ & $4(6)$ & $16(25)$ & $18(29)$ \\
\hline Enterobacter & - & - & - & $10(41.6)$ & $16(66)$ \\
\hline Citrobacter & $2(20)$ & $4(40)$ & $4(40)$ & $4(40)$ & $8(80)$ \\
\hline Acinetobacter & - & - & - & $2(33)$ & - \\
\hline
\end{tabular}

These international, enteric isolates were also very susceptible to ciprofloxacin $(55 \%$ -
$100 \%$ susceptible). Nonenteric organisms (Pseudomonas, Xanthomonas, and 
Acinetobacter) from the same international locations had overall rates of susceptibility of $47 \%$ for ciprofloxacin, and only $5 \%$ for cefepime. In our study Gram negative bacilli susceptibility ranged from $25-60 \%$ to cefepime and 29-80\% for ciprofloxacin. Thus ciprofloxacin was more effective compared to cefepime.

A worrisome trend during the last two decades has been the development of resistance to extended-spectrum cephalosporins, e.g., cefotaxime, ceftazidime, and ceftriaxone. Such resistance is most often due to the breakdown of the extended-spectrum cephalosporin by extended-spectrum $\beta$ lactamases (ESBLs), but it may also be due to plasmid-mediated or chromosomally hyperproduced AmpC (Christian et al., 2008).

Failure to detect ES $\beta$ L- and AmpC $\beta \mathrm{L}$ producing strains has contributed to their uncontrolled spread and therapeutic failures. Hence, their appearance in a hospital setting should be identified quickly so that appropriate antibiotic usage and containment measures can be implemented. For detection of various types of ES $\beta L s$, molecular methods are the best but the facilities are not available especially in the developing countries. Therefore, various phenotypic methods are recommended for routine use to detect ES $\beta \mathrm{L}$ production in Gram-negative bacilli (Sinha et al., 2008). ESBL producers were $8(8 \%)$ and $\mathrm{AmpC} \beta \mathrm{L}$ producers were $5(5 \%)$ in our study.

Resistance to fluoroquinolones is frequently observed among ESBL producers. In our study among ESBL producers $4(50 \%)$ and among AmpC producers 2(40\%) were sensitive to ciprofloxacin.

In conclusion, in summary, we found that the percentage of decreased antibiotic susceptibility across all species to drugs cefuroxime, cefotaxime and ceftazidime.The most active agents were cefepime and ciprofloxacin. Among them ciprofloxacin was more active than cefepime among all tested organisms except the Acinetobacterspp in which cefepime was more active. So considering the cost and adverse effects of cefepime and ciprofloxacin, the later can be used as first line of drug.

\section{References}

Ambler, R.P. 1980. "The structure of $\beta$ lactamases". Philos. Trans. $R$ Soc. Lond. B, 289: 321-31.

Archibald, L., $\quad$ Phillips, L., $\quad$ Monnet, D., McGowan, J.E., $\quad$ Tenover, F., Gaynes, R. 1997. "Antimicrobial resistance in isolates from inpatients and outpatients in the United States: increasing importance of the intensive care unit". Clin. Infect. Dis., 4: 211215.

Barnaud, G,. Arlet, G., Verdet, C,. Gaillot, O., Langrange, P.H., Philippon, A. 1998. "Salmonella enteritidis AmpC plasmid mediated inducible - $\beta$ lactamases (DHA-1) with an ampR gene from Morganella morganii". Antimicrob Agents Chemother, 42: 2352-8.

Ben-Ami, R., M.J. Schwaber, S. NavonVenezia, D. Schwartz, M. Giladi, I. Chmelnitsky. 2006. "Influx of extended-spectrum beta-lactamaseproducing Enterobacteriaceae into the hospital". Clin. Infect. Dis., 42: 925934.

Bertram, G., Katzung, Susan, B., Masters, Anthony, J. Trevor. "Basic and Clinical Pharmacology" 12edLange; Chapter 43: 797-800. 
Bertram, G., Katzung, Susan, B., Masters, Anthony, J. Trevor. "Basic and Clinical Pharmacology" 12edLange; Chapter 46: 834-838.

Bhattacharjee, A., Sen, M.R., Prakash, P., Gaur, A., Anupurba, S. 2008. "Increased prevalence of extended spectrum $\beta$ lactamase producers in neonatal septicaemic cases at a tertiary referral hospital". Indian J. Med. Microbiol., (serial online) (cited 2013 Aug 26);26: 356-60.

Black, J.A., Moland, E.S., Thomson, K.S. 2005. "AmpC disk test for detection of plasmid mediated AmpC $\beta$-lactamases in Enterobacteriaceae lacking chromosomal AmpC $\beta$-lactamases". $J$. Clin. Microbiol., 43: 3110-3.

Burwen, D.R., Banerjee, S.N., Gaynes, R.P. 1994. "The National Nosocomial Infections Surveillance System. Ceftazidime resistance among selected nosocomial gram-negative bacilli in the United States". J. Infect. Dis., 170: 1622-1625.

Bush, K., Jacoby, G.A., Medeiros, .AA. 1995. "A functional classification scheme for $\beta$-lactamases and its correlation with molecular structure". Antimicrob Agents Chemother., 39: 1211-33.

Christian, G., Giske, Dominique, L., Monnet, Otto Cars. 2008. "Clinical and Economic Impact of Common Multidrug-Resistant Gram-Negative Bacilli”. Antimicrob. Agents Chemother., vol. 52 no. 3 813-821.

CLSI. 2013. "Performance Standards for Antimicrobial Susceptibility Testing; Twenty Third Information Supplement". CLSI document M100 S23. Wayne, P.A: Clinical and Laboratory Standards Institute.

Cordron, P.E., Moland, E.S., Thomson, K.S. 2000. "Occurrence and detection of AmpC $\beta$-lactamases among E. coli,
Klebsiella pneumoniae and Proteus mirabilis isolates at Veterans medical centre". J. Clin. Microbiol., 38: 17916.

Håkan Hanberger, José-Angel GarciaRodriguez, Miguel Gobernado. 1999. "Antibiotic Susceptibility Among Aerobic Gram-negative Bacilli in Intensive Care Units in 5 European Countries". JAMA, 281(1): 67-71.

Itokazu, G.S., Quinn, J.P., Bell-Dixon, C. 1996. "Antimicrobial resistance rates among gram-negative bacilli recovered from patients in intensive care units: evaluation of a national post marketing surveillance program". Clin. Infect. Dis., 23: 779-784.

Jarlier, V., Fosse, T., Philippon, A. 1996. "Antibiotic susceptibility in aerobic gram-negative bacilli isolated in intensive care units in 39 French teaching hospitals" (ICU study). Intensive Care Med., 22: 1057-1065.

Jones, R.N., Marshall, S.A. 1994. "Antimicrobial activity of cefepime tested against Bush group I betalactamase-producing strains resistant to ceftazidime. A multilaboratory national and international clinical isolate study". Diag. Microbiol. Infect. Dis., 19(1): 33-38.

Livermore, D.M. 1995. "Beta-lactamases in laboratory and clinical resistance". Clin. Microbiol. Rev., 8: 557-584.

Livermore, D.M., $\quad$ Yuan, M. 1996. "Antibiotic resistance and production of extended-spectrum beta-lactamases amongst Klebsiella spp from intensive care units in Europe". J. Antimicrob. Chemother., 38: 409-424.

Paterson, D.L., and R.A. 2005. Bonomo. "Extended-spectrum betalactamases": a clinical update. Clin. Microbiol. Rev., 18: 657-686.

Philippon, A., Labia, R., Jacoby, G. 1989. "Extended-spectrum beta-lactamases". 
Antimicrob Agents Chemother., 33: 1131-1136.

Phillipon, A,. Arlet, G., Jacoby, G.A. 2002. "Plasmid determined AmpC type $\beta$ lactamases". Antimicrob Agents Chemother., 46: 1-11.

Pitout, J.D., P. Nordmann, K.B., Laupland, and L. Poirel. 2005. "Emergence of Enterobacteriaceae producing extended-spectrum beta-lactamases (ESBLs) in the community". $J$. Antimicrob. Chemother., 56: 52-59.

Shawn, R., Lockhart, Murray, A.2007. "Abramson,Susan E. Beekmann. Antimicrobial Resistance among Gram-Negative Bacilli Causing Infections in Intensive Care Unit Patients in the United States between 1993 and 2004". J. Clin. Microbiol., 45(10): 3352-3359.

Sievert, D.M., Ricks, P., Edwards, J.R., et al. 2009-10. "Antimicrobial-resistant pathogens associated with healthcare- associated infections: summary of data reported to the National Healthcare Safety Network at the Centers for Disease Control and Prevention", Infect. Control Hosp. Epidemiol., 34: 1-14.

Sinha, P., Sharma, R., Rishi, S. 2008. "Prevalence of extended spectrum beta lactamase and AmpC beta lactamase producers among Escherichia coli isolates in a tertiary care hospital in Jaipur". Indian J. Pathol. Microbiol., 51: 367-9.

Thomson, K.S. 2001. "Controversies about extended spectrum and $\mathrm{AmpC} \beta-$ lactamases". Emerg. Infect. Dis., 7: 333-6.

Verbist, L. 1991. "Incidence of multiresistance in gram-negative bacterial isolates from intensive care units in Belgium: a surveillance study". Scand J. Infect. Dis., 78(suppl): 45-53.

\section{How to cite this article:}

Vishwajith, K. Anuradha and D. Venkatesha. 2016. Comparison of Susceptibility of Gram Negative Bacilli to Cephalosporins and Ciprofloxacin. Int.J.Curr.Microbiol.App.Sci. 5(9): 205212. doi: http://dx.doi.org/10.20546/ijcmas.2016.509.023 